\title{
Ejercicio físico como intervención eficaz en el anciano frágil
}

\section{Physical exercise as an efficient intervention in frail elderly persons}

\author{
A. Casas Herrero' ${ }^{1}$ M. Izquierdo ${ }^{2}$
}

\section{RESUMEN}

El síndrome de la fragilidad define a los ancianos vulnerables que tienen un riesgo elevado de sufrir eventos adversos. Su fisiopatología y etiopatogenia es compleja, pero actualmente disponemos de medidas sencillas de capacidad funcional para su evaluación. La inactividad física, que frecuentemente asocia el envejecimiento, es uno de los factores fundamentales que contribuye a la aparición de sarcopenia, aspecto central de la fragilidad. Los programas de ejercicio físico multicomponente y, particularmente el entrenamiento de la fuerza, constituyen las intervenciones más eficaces para retrasar la discapacidad y otros eventos adversos. Así mismo, han demostrado su utilidad en otros dominios frecuentemente asociados a este síndrome como las caídas, el deterioro cognitivo y la depresión. Sin embargo, es necesario investigar cuáles son los componentes óptimos de un programa de fuerza en el frágil, así como la óptima relación dosis-respuesta que permita desarrollar guías clínicas específicas de actividad física para este grupo poblacional.

Palabras clave. Entrenamiento de fuerza. Capacidad aeróbica. Fragilidad. Envejecimiento.

\begin{abstract}
Frailty is a state of vulnerability that involves an increased risk of adverse events in older adults. It is a condition with a complex etiology and pathophysiology. At present, there are functional tools for its assessment that are simple and reliable. Physical inactivity is a major risk factor for sarcopenia, a core aspect of frailty. Currently, mulicomponent exercise programs, and especially resistance exercise, are the most relevant interventions to slow down disability and other adverse outcomes. Moreover, these programs are valuable interventions in other frailty domains such as falls, cognitive decline and depression. However, in frail aged patients it is necessary to explore optimal resistance training components and develop specific clinical guides of physical activity for this target population.
\end{abstract}

Key words. Strength training. Frailty. Aerobic capacity. Aging.
1. Division of Geriatrics. Complejo Hospitalario de Navarra. Navarra. Spain

2. Department of Health Sciences. Public University of Navarra. Navarra. Spain

Recepción: 18 de octubre de 2011

Aceptación provisional: 12 de diciembre de 2011 Aceptación definitiva: 19 de diciembre de 2011

\section{Correspondencia:}

Mikel Izquierdo, $\mathrm{PhD}$

Departamento de Ciencias de la Salud Universidad Pública de Navarra

(Campus de Tudela)

Avenida de Tarazona s/n

31500 Tudela (Navarra) SPAIN

E-mail: mikel.izquierdo@gmail.com 
FRAGILIDAD. CONCEPTO, VALORACIÓN E INDICADORES FUNCIONALES

El perfil demográfico de España ha experimentado un cambio espectacular a lo largo del pasado siglo; la población general española se duplicó, la de mayores de 65 años se multiplicó por 7 y la de octogenarios por 13. Así, hemos pasado de tener un $11,24 \%$ de personas mayores de 65 años en 1981 al $16,86 \%$ en el año $2000^{1,2}$. En dicho año, había 6.842.143 de personas mayores de 65 años y 1.545.994 mayores de 80 censadas en España. Las previsiones para la primera mitad del siglo no sólo no modifican la tendencia, sino que la confirman, situando el porcentaje de mayores de 65 años en un $20 \%$ en el año $2021^{2}$. Esto nos convertiría en el país con mayor porcentaje de persones mayores a nivel mundial en la primera mitad del siglo XXI. Estos datos justifican la necesidad de examinar el impacto del envejecimiento y el ejercicio físico sobre la salud, con el fin de prevenir sus consecuencias indeseables, mejorar el bienestar de los ancianos y facilitar su adaptación a la sociedad en que viven.

El interés sobre el envejecimiento ha crecido exponencialmente en las últimas décadas. Alguno de sus aspectos, como la discapacidad y la fragilidad, se han convertido en centro de atención de la investigación básica, clínica y poblacional. Como consecuencia de la mayor longevidad poblacional hemos asistido a un cambio en los patrones de enfermar en lo que se conoce cómo transición epidemiológica. Así la enfermedad aguda, de curso exógeno y transmisible se ha reemplazado por la edad-dependiente, origen endógeno, curso crónico y generalmente no transmisible ${ }^{3}$. En la mayoría de las ocasiones, conforme un individuo envejece (envejecimiento habitual o "usual aging") se produce un deterioro progresivo de la adaptabilidad al deteriorarse tanto la reserva funcional en múltiples niveles celulares como el control del medio interno (homeostasis). Dicha pérdida condiciona una mayor susceptibilidad a la agresión externa, al disminuir los mecanismos de respuesta y su eficacia para conservar el medio interno. Esta vulnerabilidad es el substrato fisiopatológico fundamental de la fragilidad ${ }^{3}$.

Los datos epidemiológicos del Cardiovascular Health Study demuestran que el síndrome tiene un alto impacto en la población, con una prevalencia de sujetos frágiles del $7 \%$ entre los mayores de 65 años y de prefrágiles del $47 \%{ }^{4}$. Los estudios españoles corroboran los datos americanos. Así, en el Estudio Toledo para un Envejecimiento Saludable (ETES) ${ }^{5}$ arroja una prevalencia de fragilidad del 8,4\% (mayores de 64 años) y muestra una clara relación con la edad. En el estudio FRADEA de Albacete $^{6}$ la prevalencia alcanza el 16,9\% (mayores de 69 años).

Actualmente no existe un consenso sobre cuáles son los parámetros o dominios que mejor definen el síndrome de la fragilidad. A pesar de estas imprecisiones en cuanto a definición, biología, causas y diagnóstico de la fragilidad ${ }^{7}$ existen varias certezas intuitivas, como son:

- Asociación con un riesgo incrementado de resultados adversos (caídas, anorexia-pérdida de peso, delirium, hospitalización, declive funcional, deterioro cognitivo, mortalidad, ingreso en residencia).

- Afectación de múltiples órganos como sustrato y consecuente aparición de vulnerabilidad, así como cambios que se producen en el tiempo $^{7}$.

Dos modelos han demostrado validez en el concepto y en la predicción de eventos adversos:

- Fenotipo físico de fragilidad: propuesto por Fried y col en el año $2001^{4}$ incluye los siguientes dominios: pérdida de peso no intencionada $(>4,5$ $\mathrm{kg} / \mathrm{año}$ ) debilidad (medida a través de la fuerza de prensión), cansancio, baja resistencia, lentitud (medida mediante velocidad de la marcha) y bajo grado de actividad física. Los sujetos con uno o 2 criterios se consideran pre-frágiles y aquellos con 3 o más criterios se consideran frágiles. 
- Modelo de múltiples dominios ${ }^{7}$ : postulado por diferentes autores como Rockwood o Mitniski, implica que la presencia de diversas afecciones (enfermedades, síndromes geriátricos, discapacidades y factores psicosociales) asociadas al envejecimiento se agrupan de manera aditiva para originar vulnerabilidad.

Sea cual sea el modelo y los dominios afectos en lo que sí existe consenso es en la existencia de una mayor vulnerabilidad o estado de pre-discapacidad y la predisposición a eventos adversos (discapaci- dad, institucionalización, muerte, caídas, fracturas y hospitalizaciones $)^{8}$ derivada de una pérdida de homeostasis, debido a un declive en múltiples sistemas corporales (neuromuscular, metabólico-inflamatorio, neuroendocrino, vascular) ${ }^{9}$. La consecuencia del deterioro en estos sistemas es la disminución de la reserva funcional y la aparición de sarcopenia -deterioro de la función muscular asociado al envejecimiento-, aspecto central de la fragilidad. La vía final común de este ciclo suele desembocar en la aparición de discapacidad y dependencia $^{4}$ (Fig. 1).

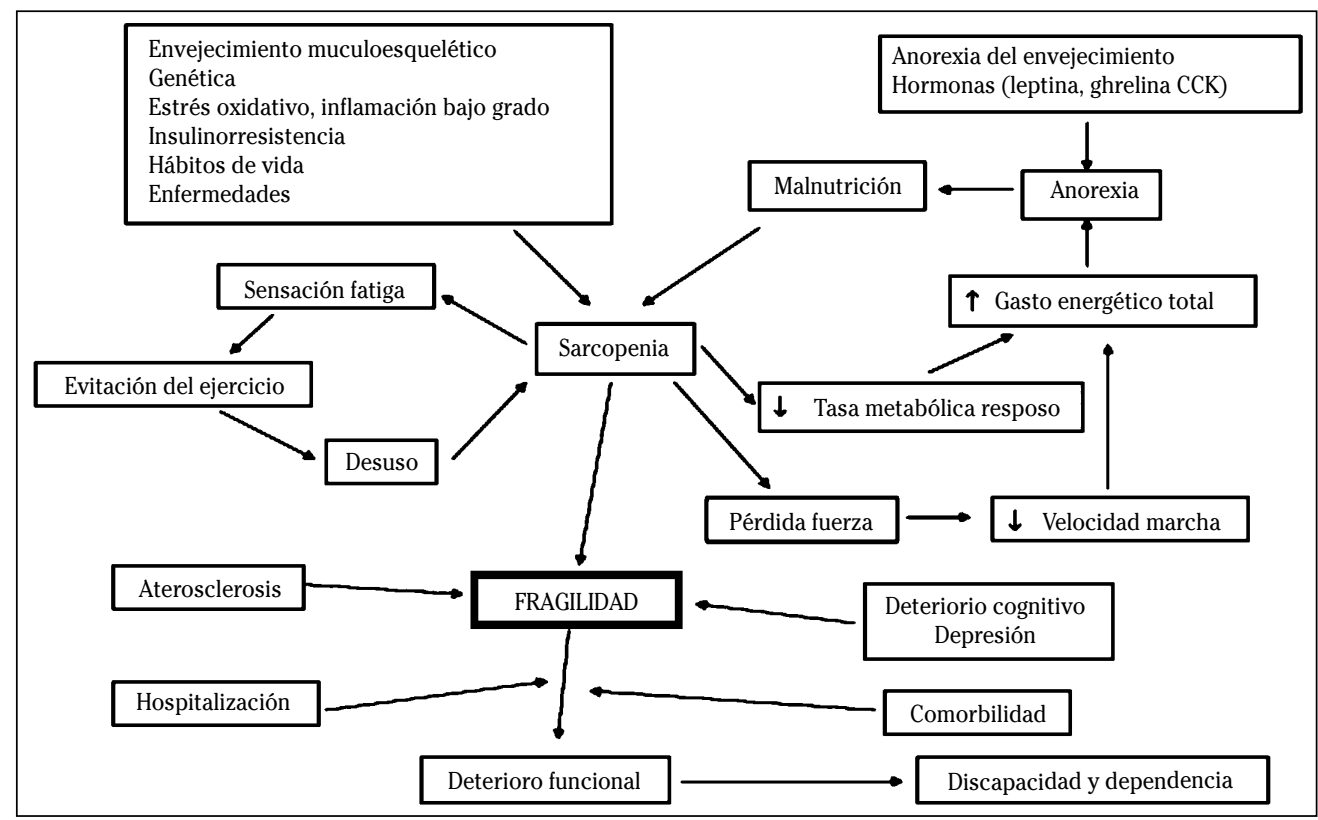

Figura 1. Ciclo de la fragilidad. Tomada de Abizanda Soler $\mathrm{P}^{29}$.

La pérdida de masa y cualidades musculares, especialmente la potencia muscular, que acontece en el envejecimiento y en la fragilidad está directamente relacionada con una reducción en la movilidad y en la capacidad de realizar las denominadas actividades básicas o instrumentales de la vida diaria (ABVD o AIVD) ${ }^{10}$. El desempeño de estas actividades está relacionada con múltiples variables (p.e. comorbilidad, regulación hormonal y función cognitiva).
No obstante, las relaciones entre parámetros de fuerza y capacidad en AVD no son lineales ${ }^{11}$, por lo que para tratar de explicar las bases etiopatogénicas de la fragilidad, las medidas de función muscular se deben acompañar de otro tipo de mediciones. Estas medidas se denominan "medidas de rendimiento o capacidad funcional" y su utilidad a la hora de cuantificar la limitación funcional hace que se hayan utilizado en numerosos estudios clínicos y epide- 
miológicos, constituyendo un instrumento fundamental en la valoración de la fragilidad y en la predicción de eventos adversos. Dentro de estas mediciones ocupan un lugar de privilegio aquellas relacionadas con la movilidad. Entre ellas podemos destacar las siguientes:

Velocidad de la marcha. Es el tiempo empleado en recorrer una distancia predeterminada, habitualmente entre 4 y 8 metros. Muchos autores consideran que puede ser una buena herramienta para detectar fragilidad $^{12}$, eventos adversos ${ }^{13}$ y supervivencia $^{14}$. Destaca por su utilidad, simplicidad y reproducibilidad en la práctica clínica diaria. Una velocidad de la marcha superior a $1,1 \mathrm{~m} / \mathrm{s}$ puede ser considerada como normal en ancianos comunitarios sin discapacidad, mientras que cuando es inferior a 0,8 $\mathrm{m} / \mathrm{s}$ detecta problemas en la movilidad y predice caídas, incluso con mayor precisión que otras pruebas funcionales ${ }^{13}$. Una velocidad menor $0,6 \mathrm{~m} / \mathrm{s}$ predice eventos adversos. Un punto de corte menor de $1 \mathrm{~m} / \mathrm{s}$ se considera un buen marcador de fragilidad ${ }^{15}$.

Time Up and Go. Desarrollado por Podsiadlo $^{16}$, comprende el tiempo invertido en levantarse de la silla sin utilizar los brazos, caminar durante 3 metros, darse la vuelta y volver a la silla y sentarse. Una puntuación inferior a 10 segundos es normal; entre 10 y 20 segundos es marcador de fragilidad y cuando es mayor de 20 segundos se considera que el anciano tiene un elevado riesgo de caídas ${ }^{8}$.

SPPB (Short Performance Battery Test). Es una herramienta eficaz para la valoración de la función física en el anciano. Combina mediciones de equilibrio (bipedestación, tándem y semitándem), marcha (velocidad de la marcha $4 \mathrm{~m}$ ), fuerza y resistencia (levantarse de la silla). Su puntuación se correlaciona de forma significativa con institucionalización y mortalidad ${ }^{17}$.

Prueba de estación unipodal. Se ha demostrado que es una herramienta útil a la hora de predecir el riesgo de caída en población anciana. Una puntuación inferior a 30 segundos se asocia con historia previa de caídas mientras que un valor superior a 30 segundos se asocia con un bajo riesgo de caída ${ }^{18}$. Recientemente, se ha observado que esta prueba se asocia con riesgo de fragilidad $^{19}$.

Fuerza de prensión en mano dominante. La pérdida de la fuerza de prensión se asocia con el envejecimiento, pero independientemente de esta relación, se ha demostrado que es un potente predictor de discapacidad, morbilidad y mortalidad y por sí solo es buen marcador de fragilidad ${ }^{20}$.

El principal objetivo en la fragilidad, una vez se ha realizado una adecuada detección de la misma, es la intervención precoz con el objetivo de prevenir el deterioro funcional y la dependencia o al menos poder ralentizar o retrasar su aparición. En un síndrome donde la etiopatogenia es compleja e intervienen múltiples vías, tiene sentido que las intervenciones sean multifactoriales. En los últimos años se han desarrollado avances en intervenciones nutricionales (suplementos proteínicos, vitamina D), farmacológicas (miméticos de la ghrelina, moduladores selectivos de los receptores androgénicos-SARM, antimiostáticos, antioxidantes y creatina) aunque la intervención que mejor resultado ha conseguido es el ejercicio físico ${ }^{21}$.

\section{ENVEJECIMIENTO Y SISTEMA NEUROMUSCULAR}

Con el envejecimiento, la capacidad funcional del sistema neuromuscular, cardiovascular y respiratorio comienza a disminuir de forma progresiva lo que conlleva un riesgo aumentado de fragilidad. Diversos estudios ${ }^{22-24}$ han observado que las personas de 75 años presentan, con respecto a los jóvenes de 20 años, una disminución de la resistencia aeróbica (45\%), fuerza de prensión (40\%), fuerza de las piernas (70\%), movilidad articular (50\%) y de la coordinación neuromuscular (90\%). La sarcopenia es uno de los principales factores que influyen en la disminución de la capacidad de mantenerse independiente en la comunidad y en la génesis de la discapacidad $^{25}$. La fuerza máxima y explosiva es necesaria para poder realizar muchas tareas de la vida diaria como subir escaleras, levantarse de una silla o pasear. Por otro 
lado, también es conocido que la reducción de la capacidad del sistema neuromuscular para generar fuerza que aparece con el envejecimiento también favorece el riesgo de caídas, típicas de este grupo de población. Además del envejecimiento "per se" uno de los factores que mejor explican la reducción de fuerza y la masa muscular asociada al envejecimiento, es la drástica reducción que se observa con el paso de los años en la cantidad y calidad de actividad física diaria. La estimación media de pérdida de masa muscular a partir de los 60 años es de $2 \mathrm{~kg}$ en varones y $1 \mathrm{~kg}$ en mujeres $^{26}$, pero sólo 10 días de reposo en cama en un anciano puede resultar en un pérdida de $1,5 \mathrm{~kg}$ de masa magra (fundamentalmente en miembros inferiores) y una disminución del $15 \%$ de la fuerza de extensión de la rodilla ${ }^{27}$. La inmovilización además induce resistencia anabólica ${ }^{28}$, disfunción mitocondrial y apoptosis ${ }^{29}$. El resultado de todo este proceso, como si se tratase de un círculo vicioso, origina que en la medida en que disminuye la práctica de actividad física diaria, disminuye la fuerza y la masa muscular lo que a su vez genera mayor sarcopenia (Fig. 2). La interrupción de este ciclo es de vital importancia para el mantenimiento de la funcionalidad de los ancianos.

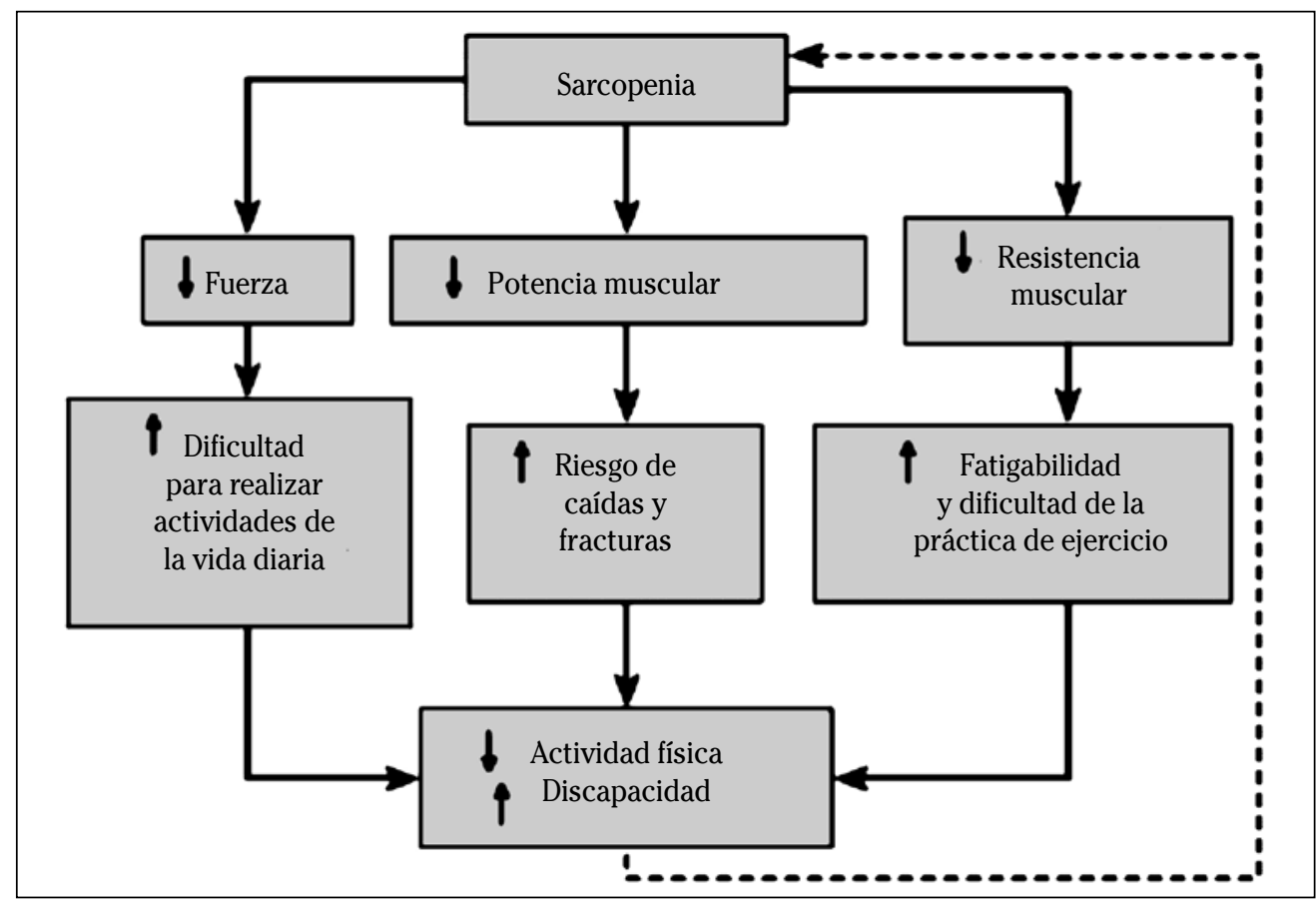

Figura 2. Modelo que explica las consecuencias funcionales de los cambios relacionados con la edad en la sarcopenia (pérdida de masa y función muscular) y el ciclo por el que se explica cómo la reducción de la actividad física acentúa el proceso de alteración. (Modificado de Hunter GR, McCarthy JP, Bamman $\mathrm{MM})^{79}$.

En las últimas décadas se ha propugnado que el entrenamiento de fuerza en personas mayores podría prevenir o retardar la pérdida de fuerza. Diversos estudios han mostrado que la realización de un entrenamiento sistemático de la fuerza máxima se acompaña de incrementos significativos en la producción de fuerza, no sólo en personas jóvenes, sino también en las mayores $^{30,31}$. Los incrementos iniciales de la fuerza pueden llegar a ser de hasta un $10-30 \%$ (o incluso más) durante las prime- 
ras semanas ó 1-2 meses de entrenamiento, tanto en personas de mediana edad como en las de edad avanzada, en ambos sexos. En los siguientes apartados se examinarán los efectos de los distintos programas de ejercicio físico en el anciano frágil.

\section{ENTRENAMIENTO DE FUERZA EN EL ANCIANO FRÁGIL}

Los programas de entrenamiento de fuerza en mayores probablemente constituyen por sí mismos la medida preventiva más eficaz para retrasar la aparición de sarcopenia y/o fragilidad ${ }^{32}$. Varios estudios y revisiones sistemáticas han demostrado que incluso en los ancianos más viejos y frágiles el entrenamiento de fuerza aumenta la masa muscular, la potencia y la fuerza muscular ${ }^{334-35}$, además de mejorar parámetros objetivos del síndrome de fragilidad tales como la velocidad de la marcha y el tiempo de levantarse de una silla ${ }^{34}$. Aunque inicialmente sus resultados sobre la mejoría de la función no eran claros, la reciente revisión sistemática realizada por Liu y Lathman ${ }^{34}$ ha demostrado que es una intervención eficaz para mejorar la función física en ancianos y retrasar por lo tanto la discapacidad, que es el principal evento adverso de la fragilidad.

La realización de un entrenamiento sistemático de fuerza máxima en mayores se acompaña de incrementos significativos en la producción de fuerza siempre y cuando la intensidad y duración del periodo de entrenamiento sean suficientes ${ }^{30,31,36}$. Los incrementos de fuerza inducidos por el entrenamiento se asocian en las primeras semanas de entrenamiento, principalmente a una adaptación en el sistema nervioso, ya sea por un aumento en la activación de la musculatura agonista o bien por cambios en los patrones de activación de la musculatura antagonista. Sin embargo, a partir de la semana 6-7 la hipertrofia muscular es un hecho evidente, aunque los cambios en los tipos de proteínas, tipos de fibras y síntesis de proteínas ocurran mucho antes.

El músculo esquelético tiene la capacidad de hipertrofiarse después de participar en un programa de entrenamiento de fuerza. La hipertrofia muscular es resultado de la acumulación de proteínas, debido a un aumento en la síntesis, reducción en la degradación o ambos. A pesar de que en los ancianos el incremento de la fuerza muscular con el entrenamiento es debido principalmente a mejoras en los patrones de activación neural, se ha demostrado que la hipertrofia muscular también contribuye a la mejora de la fuerza. Esto se ha reflejado en estudios en los que se han utilizado técnicas sensibles como la determinación del área de las fibras musculares mediante la realización de biopsias musculares, o la determinación del área de la sección transversal muscular mediante el uso de RMN o TAC ${ }^{37,38}$. Algunos estudios experimentales han mostrado en grupos de personas de edad avanzada diferencias significativas en el área de la sección transversal muscular del grupo muscular cuádriceps femoral medida antes y después de sólo 2-3 meses de un entrenamiento de fuer$\mathrm{za}^{37,39}$. Sin embargo, los efectos del entrenamiento sobre el área de la sección transversal muscular se tienen que interpretar con cautela debido a que la hipertrofia muscular inducida por el entrenamiento de fuerza puede no ser un proceso uniforme a lo largo de todo el paquete muscu$\operatorname{lar}^{40}$. Así, en un estudio realizado por Häkkinen y col $^{40}$ en mujeres de avanzada edad se observó que los cambios inducidos tras 21 semanas de entrenamiento de fuerza en el área de sección transversal determinada por resonancia magnética nuclear, no eran uniformes a lo largo de grupo muscular cuádriceps femoral, de tal forma que los aumentos fueron superiores en las regiones con más sección transversal, en las porciones proximales del vasto lateral y en las porciones distales del vasto medial. Sin embargo, estos hallazgos no se observaron en los músculos vasto intermedio y recto femoral. Por otro lado, la influencia que tiene la proporción de fibras rápidas y lentas sobre los incrementos en la fuerza muscular y área de sección transversal, en respuesta al entrenamiento de fuerza en ancianos no está clara. Así, en un grupo de personas jóvenes y mayores, los sujetos con una proporción mayor de fibras mus- 
culares rápidas mostraron mayores incrementos en el área de sección transversal de los músculos entrenados que aquellos sujetos que tenían una menor proporción de fibras rápidas ${ }^{41}$. Esto podría ser de gran importancia si la pérdida de fibras musculares que se produce con el envejecimiento realmente afectara en mayor medida a las fibras musculares de contracción rápida, tal y como han sugerido algunos estudios. No obstante, diversos estudios también han observado aumentos en el tamaño de las fibras musculares tipo I y tipo II, acompañados de una transformación desde las fibras tipo IIx hacia las de tipo Ilax y IIa $^{30}$. Sin embargo, la magnitud de la hipertrofia provocada por el entrenamiento no se correlaciona necesariamente con los incrementos observados en la fuerza máxima durante periodos de pocas semanas ${ }^{42,37}$. Esto puede explicarse por cambios a nivel del sistema nervioso y por pequeñas modificaciones en las propiedades contráctiles de las fibras con el entrenamiento.

Las adaptaciones producidas por un programa de entrenamiento de fuerza en mayores serán diferentes entre las personas y vendrán determinadas por su nivel de entrenamiento previo, situación funcional y comorbilidad asociada ${ }^{36}$. Un anciano vigoroso con funcionalidad conservada, sin comorbilidad y con un nivel de actividad física previa elevado, necesitará un tipo de entrenamiento más exigente que aquel que sea frágil y presente patologías asociadas que afecten a su función, que deberá comenzar el programa con un estímulo menor. En la actualidad, las recomendaciones realizadas por algunas instituciones y autores ${ }^{43}$ suelen ser demasiado intensas y fatigantes y no están específicamente diseñadas para el anciano frágil. Pueden inducir un aumento del riesgo de lesión, abandono y sobreentrenamiento, además de no favorecer en mayor medida el desarrollo de la fuerza y masa muscular que los efectos que pudieran surtir de utilizar intensidades inferiores. En los siguientes apartados se hará una revisión de los principales trabajos de investigación que abordan estas controversias y se presen- tarán algunas recomendaciones para la prescripción del entrenamiento de fuerza en ancianos frágiles.

\section{COMPONENTES DEL ENTRENAMIENTO PARA EL DESARROLLO DE LA FUERZA}

Las personas mayores retienen la capacidad de mejorar su fuerza muscular después de participar en un programa de entrenamiento sistemático de fuerza máxima siempre y cuando la intensidad y duración del periodo de entrenamiento sean suficientes ${ }^{36}$. Estos programas deberán seguir los mismos principios básicos de entrenamiento que los diseñados para jóvenes o deportistas: 1) principio de la sobrecarga, 2) de la progresión, 3) de la especificidad y la individualidad del entrenamiento, y 4) del desentrenamiento o reversibilidad ${ }^{44}$. Así, este tipo de programa de entrenamiento deberá producir un estímulo lo suficientemente intenso, por encima del que suponen las actividades regulares de la vida diaria, como para producir la respuesta de adaptación deseada (principio de sobrecarga), pero sin llegar a producir agotamiento o esfuerzo indebido. Una vez que el organismo se adapte a este estímulo será necesario que se modifique $\mathrm{y} / \mathrm{o}$ incremente, para que se continúe progresando (principio de la progresión). Si las cargas de entrenamiento no se incrementan progresivamente (entrenamiento de fuerza progresivo), los músculos se adaptarán al nivel de fuerza solicitado y se mantendrán los mismos niveles de fuerza hasta que no se someta al sistema neuromuscular a un estímulo mayor. Cuando una persona deja de entrenar, se producirá la regresión de las adaptaciones conseguidas. Además, el entrenamiento de fuerza deberá ser específico para los grupos musculares más utilizados y con transferencia directa (principio de especificidad) a actividades de la vida diaria como, por ejemplo, sostener una bolsa de la compra o subir escaleras.

Las adaptaciones producidas por un programa de entrenamiento de fuerza serán diferentes entre las personas y vendrán 
determinadas por su nivel de entrenamiento previo y edad. Una persona que se encuentre en buen estado de forma necesitará un tipo de entrenamiento más exigente que aquel que sea inactivo y deba comenzar el programa de entrenamiento con un estímulo menor.

La efectividad y resultado de un entrenamiento para el desarrollo de la fuerza depende de la aplicación de una carga adecuada, es decir, de factores como la intensidad, volumen de entrenamiento (series por repeticiones), frecuencia y tipología de los ejercicios recomendados (isocinético/ resistencia variable/isoinercial), periodos de recuperación entre las series y la frecuencia de entrenamiento. Diferentes combinaciones de las variables que componen el entrenamiento, como por ejemplo el número de repeticiones por serie, número de series y descanso entre series, originan diferentes respuestas fisiológicas. De manera general, todos los programas de entrenamiento inducen ciertas mejoras de la fuerza máxima, hipertrofia o potencia muscular. Sin embargo, determinadas combinaciones tendrán un especial énfasis de adaptación en unas o en otras manifestaciones de la fuerza. Por ejemplo, en el trabajo realizado por Kraemer y col $^{45}$ se observó que 3 series de 10 repeticiones máximas (10RM) con 1 minuto de descanso entre series aumentaba significativamente la concentración de lactato y la hormona del crecimiento en comparación con la realización de 3 series a una intensidad de 5RM con 3 minutos de descanso. Por tanto, según estos estudios parece evidente que si el objetivo del entrenamiento es desarrollar la capacidad de tolerar la acidosis láctica y aumentar la hipertrofia muscular, este tipo de diseño de entrenamiento será el más efectivo. En los siguientes apartados se especificarán estas características para el desarrollo de la fuerza máxima con la influencia de tipo neural o hipertrófica y el desarrollo de la potencia muscular.

\section{INTENSIDAD}

La intensidad de un estímulo es el grado de esfuerzo que exige un ejercicio, en el entrenamiento con cargas. Viene representado por el peso que se utiliza en términos absolutos o relativos, así como por el número máximo de repeticiones que se pueden realizar con un determinado peso $^{43,46,47}$. En función del número de repeticiones que se pueden realizar con una carga determinada hasta la fatiga se producen diferentes efectos sobre la fuerza. Clásicamente se ha comentado que el desarrollo de la fuerza máxima se consigue más eficazmente con cargas elevadas y pocas repeticiones máximas (RM) (desde 4RM-10RM), mientras que si se reduce la resistencia y se aumenta el número de repeticiones (12RM-20RM) se favorecerá el desarrollo de la resistencia muscular ${ }^{42,44}$. A efectos prácticos, el porcentaje de la fuerza dinámica máxima (\% de 1RM) correspondiente al peso con el que se podrían efectuar un máximo de 8 a 12 repeticiones se encuentra, aproximadamente, entre el $70-80 \%$. La zona de 15 a 20 repeticiones corresponde a un 50-60\% de 1RM. Para población anciana las recomendaciones del Colegio Americano de Medicina del Deporte en cuanto a la intensidad son inferiores, de 10-15RM (65-75\%) aunque no existen recomendaciones específicas para los frágiles ${ }^{44}$. Sin embargo, cada vez parece más evidente que, la realización de repeticiones hasta el fallo con estas intensidades pueden suponer un excesivo e innecesario esfuerzo, además de ser perjudiciales para la salud y el rendimiento, no sólo para estos grupos de poblaciones especiales (p.e. envejecimiento, obesidad, diabetes), sino también para la mayoría de los deportistas. Por otro lado, existe poca evidencia que muestre el efecto superior de estas intensidades sobre otras más inferiores (p.e. 20RM-30RM) en personas previamente no entrenadas o en deportes con necesidades medias de fuerza ${ }^{47-50}$. Una revisión reciente ${ }^{, 51}$, que analiza cuáles deben ser las intensidades del entrenamiento de fuerza en población anciana (no se especifica si frágil), concluye que intensidades elevadas son superiores a las bajas en términos de mejoría de fuerza máxima pero no necesariamente en términos de ganancias funcionales. 


\section{POTENCIA MUSCULAR}

La potencia es el producto de la fuerza y de la velocidad, es decir la situación en la que se obtiene el máximo rendimiento muscular. Cuando la resistencia a vencer es ligera, la fuerza máxima tiene poca importancia en la producción de potencia pero su influencia aumenta a medida que se incrementa la resistencia. Durante el envejecimiento, la potencia se deteriora más rápida y precozmente que la fuerza ${ }^{22,23}$. Es más, la potencia muscular tiene una relación más significativa con la capacidad funcional del anciano que la fuerza ${ }^{52}$. Además del efecto del envejecimiento sobre la potencia muscular, existen cambios a nivel del sistema nervioso, como el deterioro en la activación neuromuscular voluntaria, que pueden contribuir a reducir la potencia $^{53}$. En los ancianos (incluso en los más viejos) se puede mejorar la potencia mediante el entrenamiento al $60 \%$ de $1 R M$ y con la máxima velocidad a esta resistencia (p.e. tan rápido como sea posible) que estará entre el 33-60\% de la velocidad máxima sin resistencia ${ }^{53,54}$.

Estudios realizados en la década pasada han demostrado en el anciano que, el entrenamiento combinado de fuerza máxima y potencia muscular con duraciones desde 10 hasta 48 semanas, permite mejorar significativamente la fuerza máxima dinámica ${ }^{30}$. Estudios en ancianos jóvenes no frágiles (media de 64 años) comparativamente con adultos de mediana edad, han objetivado similares mejorías en términos de potencia muscular tras cuatro meses de entrenamiento, lo que supone recuperar hasta 20 años de edad funcional en términos de potencia muscular ${ }^{31}$.

En relación a lo expuesto anteriormente, podría plantearse que el entrenamiento basado en la potencia muscular (altas velocidades) podría ser más beneficioso en términos de mejorías funcionales que los programas de resistencia (bajas velocidades). Un metanálisis reciente ${ }^{52}$ que revisa 11 estudios y analiza esta hipótesis, llega a la conclusión que parece haber beneficios funcionales en entrenamientos a altas velocidades, pero no resultan clí- nicamente relevantes. La totalidad de los estudios se realizaron en ancianos no frágiles, por lo que los autores recomiendan su prescripción en ancianos "sanos" ya que además, no se pudieron sacar conclusiones sobre su seguridad. Por lo tanto, en el anciano frágil, parece lo más razonable recomendar un programa de entrenamiento de fuerza con velocidades bajas y moderadas.

\section{VOLUMEN Y FRECUENCIA DE ENTRENAMIENTO}

El volumen de entrenamiento es una medida de la cantidad total de ejercicio efectuado. Se expresa en función del número de repeticiones, kilogramos totales levantados, o duración de la sesión o período de entrenamiento. Clásicamente, los programas de entrenamiento para el desarrollo de la fuerza recomiendan realizar tres series de $6-12$ repeticiones, durante 3 días a la semana.

Una de las controversias en el entrenamiento de fuerza se deriva del volumen de entrenamiento utilizado. Los estudios experimentales parecen indicar que no se puede aceptar que cuanto más volumen se pueda realizar mejor será el resultado. Esta controversia se ha centrado en el debate relacionado con la utilización de una o más series por ejercicio. Existen propuestas que indican que los programas que utilizan una serie por ejercicio obtienen incrementos de parecida magnitud que aquellos que utilizan múltiples series, mientras otros han mostrado que los programas que utilizan múltiples series obtienen incrementos superiores. Las discrepancias en los resultados pueden venir explicadas por las distintas características de los sujetos a los que se les somete al entrenamiento de la fuerza. Esto significa que es probable que las personas principiantes respondan de manera favorable a una o múltiples series por ejercicio, especialmente durante las semanas iniciales de entrenamiento, mientras que en las personas entrenadas los programas que utilizan múltiples series sean los que proporcionan mejoras superiores en el desarrollo de la fuerza. 
Estudios más recientes revelan que la capacidad de adaptación con el entrenamiento de fuerza parece ser menor en las personas mayores que en los jóvenes ${ }^{55}$. Así mismo, cuando la intensidad y o la frecuencia de entrenamiento aumenta, disminuye la capacidad de adaptación en la mejora de la fuerza, especialmente en los grupos de edad avanzada ${ }^{22}$. En consecuencia, es posible que con personas mayores y especialmente los más frágiles se deba ser más conservador en la progresión de las diferentes variables relacionadas con el entrenamiento (volumen, intensidad y frecuencia) y que los diseños de entrenamiento deban ser diferentes a los utilizados con personas más jóvenes. El Colegio Americano de Medicina del Deporte ${ }^{46}$ sugiere que en personas de mediana edad y edad avanzada, el entrenamiento de fuerza para mejorar la condición física general debe realizarse con una frecuencia de 2-3 sesiones por semana aunque no hace una referencia específica para el anciano frágil. En esta línea, considera que una serie de entrenamiento es más eficaz que múltiples series ya que permite mejorar la fuerza casi en igual magnitud que un entrenamiento con múltiples series ${ }^{56}$. Este tipo de programas necesitan menos tiempo para su realización y producen beneficios similares sobre la salud y el estado de forma en personas mayores previamente inactivas. Las recomendaciones de la Sociedad Americana de Geriatría ${ }^{57}$ y de la Sociedad Americana del Corazón ${ }^{58}$ tampoco son específicas para el anciano frágil.

En resumen, las recomendaciones realizadas en la actualidad por algunas instituciones y autores en el ámbito del entrenamiento de fuerza y potencia muscular, se alejan de la realidad. Este tipo de recomendaciones suelen ser demasiado intensas y fatigantes y pueden inducir un aumento del riesgo de lesión y sobreentrenamiento, además de no favorecer en mayor medida el desarrollo de la fuerza y masa muscular que los efectos que pudieran surtir de utilizar intensidades inferiores. La creencia más generalizada, especialmente en la literatura científica americana, es que para mejorar la fuerza máxima hay que realizar repeticiones por serie hasta el fallo (p.e. 8/10/12 repeticiones máximas RM) Sin embargo, diferentes estudios muestran que realizar repeticiones hasta el fallo no es necesario y puede incluso producir sobreentrenamiento $\mathrm{y}$ lesiones por sobrecarga $(47,48,50)$. Como aplicación práctica de estos trabajos se sugiere que el entrenamiento de fuerza, cuando se realiza en personas sedentarias o de edad avanzada, especialmente si son frágiles debería comenzar realizando 8-10 repeticiones por serie con un peso que pudiésemos realizar 20 repeticiones máximas (20 RM) o más y no sobrepasar la realización de 4-6 repeticiones por serie con un peso que pudiésemos realizar 15 RM.

\section{ENTRENAMIENTO COMBINADO DE FUERZA Y RESISTENCIA}

Durante las últimas décadas se ha prestado una especial atención a la combinación del entrenamiento de fuerza muscular y resistencia aeróbica. Los resultados de estos trabajos muestran que entrenamientos de 10 a 12 semanas de duración, con una frecuencia semanal comprendida entre 4 y 11 sesiones, a intensidades comprendidas entre el 60 y el $100 \%$ de $\mathrm{VO}_{2}$ máx en bicicleta, y a intensidades comprendidas entre el 40 y el $100 \%$ de 1 RM en el trabajo de fuerza, se acompañaron de un aumento del 6 al 23\% del $\mathrm{VO}_{2}$ máx y del 22 al $38 \%$ de la fuerza máxima ${ }^{59}$. En la mayoría de estos trabajos, la magnitud del incremento observado en la fuerza máxima del miembro inferior fue superior en el grupo que realizaba exclusivamente el entrenamiento de fuerza máxima, que la observada en el grupo que realizaba un programa combinado de fuerza y resistencia aeróbica. Los mecanismos que pueden explicar la inhibición del desarrollo de la fuerza muscular después de participar en un programa combinado de fuerza y resistencia, en comparación cuando sólo se realiza un programa de entrenamiento de fuerza, no están del todo definidos aunque se postulan determinadas hipótesis como el sobreentrenamiento y la falta de adaptación metabólica y morfológica del mús- 
culo esquelético al entrenamiento combinado $^{60}$. En ancianos y particularmente en frágiles son poco conocidos los efectos de un programa combinado de fuerza y resistencia aeróbica. La mayoría de estos trabajos se han realizado en ancianos sanos y muestran que las mejoras observadas en la fuerza máxima del miembro inferior en el grupo que realiza un entrenamiento exclusivo de fuerza no son diferentes a las observadas en el grupo que realiza un programa combinado de fuerza y resistencia ${ }^{47}$. No obstante, recientemente estudios realizados en población frágil que combinan actividad aeróbica y ejercicio de fuerza, han demostrado mejorías en los parámetros funcionales de la fragilidad tales como la velocidad de la marcha y $\mathrm{SPPB}^{61,62}$.

La mayoría de los trabajos que han estudiado los efectos de un programa combinado de fuerza y resistencia lo han realizado examinando el efecto de la combinación de ambas cualidades (fuerza y resistencia) en la misma sesión, sin embargo no se conocen estudios que hayan analizado el efecto de sustituir una sesión de fuerza por una de resistencia o viceversa. En un trabajo realizado en nuestro laboratorio se examinó en 31 hombres sanos (65-74 años) el efecto del entrenamiento (2 veces por semana durante 16 semanas) exclusivo de fuerza (S), exclusivo de resistencia (E) o combinado (SE) (1 sesión/semana $\mathrm{S}+1$ sesión/semana E) sobre la fuerza máxima del miembro inferior, el área de sección transversal del cuádriceps femoral y la potencia máxima alcanzada durante un test progresivo hasta el agotamiento en cicloergómetro (WL máx). La fuerza de la extremidad inferior y el área de sección transversal del cuádriceps femoral en $\mathrm{S}$ (41 y $11 \%$, respectivamente) y SE (38 y $11 \%$, respectivamente) fueron mayores que los registrados en E (11 y 4\%, respectivamente).Los aumentos observados en WL máx fueron mayores en SE (28\%) y E (23\%) que en $\mathrm{S}(10 \%)$. Un resultado interesante de este trabajo fue la ausencia de diferencias significativas entre el entrenamiento exclusivo de fuerza y el entrenamiento combinado en los incrementos de fuerza y área de sección transversal. El incremento de potencia máxima en el test incremental fue similar con el entrenamiento exclusivo de resistencia y el entrenamiento combinado. Estos resultados sugieren que un programa de entrenamiento combinado de fuerza y resistencia en personas mayores produce incrementos similares en la fuerza y la masa muscular que un programa exclusivo de entrenamiento de fuerza e incrementos similares en la potencia máxima aeróbica que los cambios producidos por un programa exclusivo de entrenamiento de la resistencia cardiovascular ${ }^{47}$.

\section{PROGRAMA DE EJERCICIO FÍSICO MULTICOMPONENTE}

Tradicionalmente se conoce a los programas que engloban ejercicios de resistencia, flexibilidad, equilibrio y fuerza. Existen dos revisiones sistemáticas recientes que analizan el beneficio de estos programas en frágiles. En la revisión de Chin y col $^{63}$ examinaron el efecto del ejercicio en la capacidad funcional de los ancianos frágiles. Su conclusión principal es que tanto los programas de fuerza como los multicomponentes eran intervenciones que mejoraban la capacidad funcional de esta población. Posteriormente Daniels y $\mathrm{col}^{64}$ analizaron las intervenciones que prevenían discapacidad en ancianos frágiles de la comunidad. Los estudios de intervención de ejercicio revisados mostraron una mayor superioridad de los programas multicomponentes frente al entrenamiento de fuerza aislado de la extremidad inferior, particularmente en los moderadamente frágiles. Aquellas intervenciones cuya duración era mayor ( $>5$ meses), con una frecuencia de 3 veces por semana, 30-45 minutos - sesión, parece que mostraban una mayor beneficio en términos funcionales. Hay que destacar que de los 4.062 estudios seleccionados sólo 10 cumplieron criterios de inclusión por problemas metodológicos en cuanto a criterios de inclusión, fundamentalmente porque no se especificaba qué criterios usaban para definir fragilidad. 


\section{EFECTOS DEL EJERCICIO FÍSICO SOBRE LOS DOMINIOS DE LA FRAGILIDAD}

El principal evento adverso de la fragilidad es el deterioro funcional y la discapacidad y dependencia (Fig. 1). Como ya ha quedado reseñado en diversos estudios epidemiológicos ${ }^{65,66}$ la práctica regular de ejercicio físico se asocia con una disminución del riesgo de discapacidad para ABVD No obstante, todavía no está del todo aclarado si la actividad física puede prevenir o revertir la fragilidad. En un reciente estudio observacional ${ }^{67}$ llevado a cabo en 2.500 ancianos (donde se definió fragilidad por una Velocidad de la Marcha (VM) $<0,6 \mathrm{~m} / \mathrm{s}$ y la incapacidad de levantarse sin ayuda una la silla) observaron que aquellos ancianos con una actividad física regular, tenían menor probabilidad de desarrollar fragilidad. Además existía 3 veces más probabilidad de presentar fragilidad severa en sedentarios frente activos. La transición desde estadios leves de fragilidad a estadios severos era mucho más frecuente en sedentarios.

Actualmente el concepto de fragilidad es muy amplio y dinámico (Fig. 1) y engloba otros dominios que están interrelacionados tanto en su etiopatogenia como en su vulnerabilidad para padecer eventos adversos. Cabe destacar los siguientes, en los que el ejercicio físico puede constituir una intervención predominante:

- Caídas. Habitualmente interrelacionadas con el síndrome de fragilidad constituyendo un motivo de consulta y evento adverso extraordinariamente frecuente en el paciente frágil. Su abordaje resulta complejo y las intervenciones habitualmente deben ser multifactoriales. El ejercicio físico quizás sea la intervención más probada y testada en la prevención de caídas. Es conocido que resulta eficaz para reducir el riesgo y la tasa de caídas tanto en población comunitaria como residencial $^{68,69}$. Los ejercicios en grupo multicomponente (equilibrio, fortalecimiento, fuerza y resistencia) y el Tai Chi como ejercicio grupal parecen reducir la tasa y el riesgo de caídas y son especialmente beneficiosos en población anciana frágil con caídas ${ }^{70}$.

- Deterioro cognitivo. La relación entre el deterioro cognitivo y la fragilidad es íntima y probablemente biyectiva ya que comparten bases fisiopatológicas comunes y resultados a corto y medio plazo (hospitalización, caídas, discapacidad, institucionalización y mortalidad $)^{71}$. Esta relación se pone de manifiesto porque probablemente el sistema nervioso central y muscular comparta vías patogénicas comunes en el devenir de la discapacidad. En el estudio Toledo de envejecimiento y fragilidad ${ }^{71}$, se ha observado como el deterioro cognitivo y la fuerza mantienen un relación directamente proporcional. La demencia comparte parcialmente los síntomas que forman parte del fenotipo de fragilidad como es la disminución de la velocidad de la marcha y la disminución de la actividad. Algunos autores consideran que incluso ambos síndromes se pueden englobar dentro de una misma entidad clínica. En este sentido tiene lógica que aquellas intervenciones que resultaran eficaces en el paciente frágil pudiesen ser beneficiosas en el anciano con deterioro cognitivo y viceversa. Estudios recientes como el de Liu-Ambrose y $\mathrm{col}^{72}$ han demostrado cómo programas de ejercicio de resistencia semanales durante 12 semanas, en una cohorte de ancianas, no solo provocan aumentos de la velocidad de la marcha, sino que resultan beneficiosos en la mejoría de funciones cognitivas ejecutivas, que están interesantemente relacionadas con el riesgo de caída ${ }^{73}$. De tal forma que, un posible mecanismo que explique la disminución del riesgo de caída en pacientes frágiles con deterioro cognitivo, puede radicar en la mejoría de las funciones ejecutivas mediada por el ejercicio físico. En un análisis secundario este grupo de autores han corroborado esta hipótesis ${ }^{74}$, mostrando cómo mejorías en la función ejecutiva se asocian con incrementos en la velocidad de la marcha y en la fuerza muscular del cuádriceps.

- Depresión. Es incluida por muchos autores dentro del espectro de la fragilidad. El fenotipo de fragilidad descrito por Fried (pérdida de peso no intencionada, 
debilidad, disminución de actividad física, cansancio, lentitud) puede ser típico de un cuadro depresivo del anciano. Además también comparten bases etiopatogenias inflamatorias-inmunológicas ${ }^{75}$. Es conocido que el ejercicio físico mejora los síntomas depresivos a corto plazo, fundamentalmente en aquellos que están ya deprimidos ${ }^{76}$. No obstante, sus efectos a largo plazo sobre síntomas depresivos y ansiosos en ancianos frágiles queda por clarificar en ensayos clínicos. Una de las posibles hipótesis que explica este potencial efecto antidepresivo y ansiolítico radica en las propiedades antiinflamatorias del ejercicio físico ${ }^{77}$.

Entre los problemas frecuentes del ejercicio físico en ancianos frágiles, se encuentran los relacionados con la comorbilidad, aspecto que con gran frecuencia está presente y se correlaciona con el síndrome de la fragilidad. Por sí misma no contraindica un programa de ejercicio pero sí requiere una evaluación médica cuidadosa previa al comienzo del programa. En general las contraindicaciones absolutas suelen ser cardiovasculares (infarto cardiaco reciente o angina inestable, hipertensión no controla$\mathrm{da}$, insuficiencia cardiaca aguda y bloqueo $\mathrm{AV}$ completo ${ }^{78}$.

Los principales riesgos del entrenamiento aeróbico y de fuerza se resumen en la Tabla 1 donde se muestran principios generales, recomendaciones, beneficios y riesgos de ambos programas de ejercicio. Como se ha señalado previamente, en población anciana frágil se recomiendan programas más conservadores en cuanto a intensidades, potencia, volumen y frecuencia de entrenamiento. Cuanto más gradual sea la progresión mejor será la tolerancia y se minimizarán efectos secundarios. Hay que tener en cuenta que en muchos estudios no se señalan adecuadamente la aparición de efectos secundarios ${ }^{34}$. Por último, es destacable la adherencia como un problema muy habitual en los programas de ejercicio en ancianos frágiles que habitualmente no han realizado actividad física previa. Generalmente es mejor en ejercicios aeróbicos (caminar, bicicleta) frente a programas de fuerza y en ejercicios grupales frente a los realizados en domicilio.

Tabla 1. Principios generales, recomendaciones, beneficios y riesgos de un programa de entrenamiento aérobico y de fuerza (Adaptada de Landi y col) ( $^{78}$

\begin{tabular}{|l|l|l|}
\hline \multicolumn{1}{|c|}{ Entrenamiento aeróbico } & \multicolumn{1}{|c|}{ Entrenamiento de fuerza } \\
\hline Principios generales & $\begin{array}{l}\text { Grandes grupos musculares } \\
\text { Muchas repeticiones } \\
\text { Baja resistencia }\end{array}$ & $\begin{array}{l}\text { Contracción varios grupos musculares } \\
\text { Pocas repeticiones } \\
\text { Moderada-alta resistencia }\end{array}$ \\
\hline Recomendaciones & $\begin{array}{l}\text { Ejercicio aeróbico bajo impacto } \\
\text { Comienzo baja intensidad y corta } \\
\text { duración (5 minutos) } \\
\text { Calentamiento y estiramiento }\end{array}$ & $\begin{array}{l}\text { Medir fuerza y potencia basal } \\
\text { Carga inicial 40-50\% } \\
\text { Grandes músculos pareados (ag-antag) } \\
\text { Pequeños incrementos carga }\end{array}$ \\
\hline Beneficios & $\begin{array}{l}\text { CV, composición corporal, metabólico } \\
\text { Resistencia muscular } \\
\text { Comorbilidad }\end{array}$ & $\begin{array}{l}\text { 个 fuerza, potencia, masa muscular } \\
\text { magra } \\
\text { Rango de movilidad (flexibilidad) } \\
\text { Función física }\end{array}$ \\
\hline Riesgos & $\begin{array}{l}\text { Evento cardiaco } \\
\text { Daño musculoesquelético }\end{array}$ & $\begin{array}{l}\text { Lesión muscular } \\
\text { Fracturas, exacerbación enf. articular }\end{array}$ \\
\hline
\end{tabular}

En resumen, de manera general, la práctica de ejercicio físico es la intervención más eficaz para retrasar la discapacidad y los eventos adversos que asocia habitualmente el síndrome de la fragilidad. El entrenamiento de fuerza, en particular, cada vez tiene más resultados favorables en este grupo poblacional y sus efectos son más destacados en otros dominios del síndrome como las caídas y el deterioro cognitivo. En la actualidad, son necesarios más estudios aleatorizados que aclaren la 
utilización óptima de los componentes de un programa de fuerza y si éstos resultan más beneficiosos en términos funcionales que los multicomponente. En este contexto, se recomienda desarrollar guías clínicas específicas para pautar ejercicio físico en el anciano frágil

\section{Agradecimientos}

Este trabajo se ha realizado en parte gracias a los proyectos de investigación del Ministerio de Salud, Instituto de Salud Carlos III, Departamento de Salud del Gobierno de Navarra, Consejo Superior de Deportes y Ministerio de Economía y Competitividad I+D+I (2008-2011) del de España (RD06/013/1003 and 87/2010, 008/EPB10/11 y DEP2011-24105), respectivamente.

\section{BIBLIOGRAFÍA}

1. CASSEL CK. Use it or lose it: activity may be the best treatment for aging. JAMA 2002; 288 : 2333-2335.

2. Morley JE. The top 10 hot topics in aging. $\mathbf{J}$ Gerontol A BiolSci Med Sci 2004; 59: 24-33.

3. De la Fuente Gutiérrez C. Fundamentos demográficos y biomédicos para una atención sanitaria específica al anciano. En: Leocadio Rodríguez-Mañas, Juan José Solano Jaurrieta. Bases de la atención sanitaria al anciano. Sociedad Española de Medicina Geriátrica. Madrid, 2001: 15-55.

4. Fried LP, Tangen CM, Walston J, Newman AB, Hirsch C, Gottdiener J, Seeman T et al. Cardiovascular Health Study Collaborative Research Group. Frailty in older adults: evidence for a phenotype. J Gerontol A Biol Sci Med Sci 2001; 56: M146-155.

5. García-García FJ, Gutiérrez Ávila G, Alfaro Acha A, Amor Andres MS, De los Angeles de la Torre Lonza M, Escribano Aparicio MV et al. The prevalence of frailty syndrome in an older population from Spain. The Toledo Study for Healthy Aging. J Nutr Health Aging 2011; 15 : 852-856.

6. Abizanda Soler P, López-Torres Hidalgo J, RomeRo Rizos L, López Jiménez M, SÁnchez JuRado PM, AtIEnZAR NúÑEz $P$ et al. Frailty and dependence in Albacete (FRADEA study): reasoning, design and methodology. Rev Esp Geriatr Gerontol $2011 ; 46$ : 81-88.
7. Rockwood K, Mitnitski A. Frailty in relation to the accumulation of deficits. J Gerontol A Biol Sci Med Sci 2007; 62: 722-727.

8. Abizanda Soler P, Gómez-Pavón J, Martín LesenDE I, BAZTÁN CORTÉS JJ. Frailty detection and prevention: a new challenge in elderly for dependence prevention. Med Clin (Barc) 2010; 135: 713-719.

9. Bergman H, Ferrucci L, Guralnik J, Hogan DB, Hummel S, Karunananthan S ET al. Frailty: an emerging research and clinical paradigm-issues and controversies. J Gerontol A Biol Sci Med Sci 2007; 62: 731-737.

10. Rantanen T, Guralnik JM, Izmirlian G, Williamson JD, SimONSick EM, FERrucCi L et al. Association of muscle strength with maximum walking speed in disabled older women. Am J Phys Med Rehabil 1998; 77: 299-305.

11. Jette AM, Jette DU. Functional and behavioral consequences of sarcopenia. Muscle Nerve Suppl 1997; 5: S39-S41.

12. Abellan van Kan G, Rolland Y, Houles M, GiLlette-Guyonnet S, Soto M, Vellas B. The assessment of frailty in older adults. Clin Geriatr Med 2010; 26 : 275-286.

13. Montero-Odasso M, Schapira M, Soriano ER, VARela M, Kaplan R, CAMERA LA et al. Gait velocity as a single predictor of adverse events in healthy seniors aged 75 years and older. J Gerontol A Biol Sci Med Sci 2005; 60:13041309.

14. Studenski S, Perera S, Patel K, Rosano C, FaulKNER K, INZITARI M ET AL Gait speed and survival in older adults. JAMA 2011; 305: 50-58.

15. Cesari M, Kritchevsky SB, Penninx BW, Nicklas BJ, Simonsick EM, Newman AB et al. Prognostic value of usual gait speed in well-functioning older people-results from the Health, Aging and Body Composition Study. J Am Geriatr Soc 2005; 53: 1675-1680.

16. Podsiadlo D, Richardson $S$. The "time up and go test": a test of basic functional mobility for frail elderly persons. J Am Geriatr Soc 1991; 39: 142-148.

17. Guralnik JM, Simonsick EM, Ferrucci L, Glynn RJ, Berkman LF, Blazer DG et al. A short physical performance battery assessing lower extremity function: association with self-reported disability and prediction of mortality and nursing home admission. J Gerontol 1994; 49: M85-94.

18. Hurvitz EA, Richardson JK, Werner RA, RuHL AM, DIXON MR. Unipedal stance testing as an indicator of fall risk among older outpatients. Arch Phys Med Rehabil 2000; 81: 587-591. 
19. Martínez-Ramírez A, Lecumberri P, Gómez M, RoDRIGUEZ-MaÑAS L, GARCíA FJ, IzQUIERDO M. Frailty assessment based on wavelet analysis during quiet standing balance test. J Biomech 2011; 44: 2213-2220.

20. Syddall H, Cooper C, Martin F, Briggs R, Ainie SaYER A. Is grip strength a useful single marker of frailty? Age Ageing 2003; 32: 650-656.

21. Abizanda Soler P. Update on frailty. Rev Esp Geriatr Gerontol 2010; 45: 106-110.

22. HÄKKinen K, M Alen, M Kaldinen, M Izquierdo, K JoKelainen, H LASSILA et al. Muscle CSA, force production, and activation of leg extensor muscles during isometric and dynamic actions in middle-aged and elderly men and women. J Aging Phys Act 1998a; 6: 232-247.

23. Izquierdo M, Ibáñez J, Gorostiaga E.M, Garrués M, ZÚÑIGA A, ANTón A et al. Maximal strength and power characteristics in isometric and dynamic actions of the upper and lower extremities in middle-aged and older men. Acta Physiol Scand 1999; 167: 57-68.

24. Izquierdo M, Aguado X, González R, López JL, HÄKKINEN K. Maximal and explosive force production capacity and balance performance in men of different ages. Eur J Appl Physiol Occup Physiol 1999; 79: 260-267.

25. Cruz-Jentoft AJ, Baeyens JP, Bauer JM, Boirie Y, Cederholm T, Landi F et al. European Working Group on Sarcopenia in Older People. Sarcopenia: European consensus on definition and diagnosis: Report of the European Working Group on Sarcopenia in Older People. Age Ageing 2010; 39: 412-423.

26. Janssen I, Heymsfield SB, Wang ZM, Ross R. Skeletal muscle mass and distribution in 468 men and women aged 18-88 yr. J Appl Physiol 2000; 89: 81-88.

27. Kortebein P, Ferrando A, Lombeida J, Wolf R. Effect of 10 days of bed rest on skeletal muscle in healthy older adults. JAMA 2007; 297: 1772-1774.

28. Glover EI, Phillips SM, OAtes BR, Tang JE, TARnoplsky MA, SElby A et al. Immobilization induces anabolic resistance in human myofibrillar protein synthesis with low and high dose amino acid infusion. J Physiol 2008; 586: 6049-6061.

29. Marzetti E, Leeuwenburgh C. Skeletal muscle apoptosis, sarcopenia and frailty at old age. Exp Gerontol 2006; 41: 1234-1238.

30. HÄKKinen K, Kallinen, M, Izquierdo M, Jokelainen $\mathrm{K}$, Lassila H, MälkiÄ E et al. Changes in agonist-antagonist EMG, muscle CSA, and force during strength training in middle-aged and older people. J Appl Physiol. 1998; 84: 13411349.

31. Izquierdo M, HäKkinen K, Antón A, Garrues M, IBAÑEz J, Gorostiaga EM et al. Effects of strength training on muscle power and serum hormones in middle-aged and older men. J Appl Physiol 2001; 90: 1497-1507.

32. Rolland Y, Dupuy C, Abellan van Kan G, Gillette S, Vellas B. Treatment Strategies for sarcopenia and frailty Med Clin N Am 2011; 95: 427 438.

33. Latham NK, Bennett DA, Stretton CM, Anderson CS. Systematic review of progressive resistance strength training in older adults. J Gerontol A Biol Sci Med Sci 2004; 59: 48-61.

34. Liu CJ, Latham NK. Progressive resistance strength training for improving physical function in older adults. Cochrane Database Syst Rev 2009 CD002759.

35. Hasten DL, Pak-Loduca J, Obert KA, YarashesKI KE. Resistance exercise acutely increases MHC and mixed muscle protein synthesis rates in 78-84 and 23-32 yr old. Am J Physiol Endocrinol Metab 2000; 278: E620-E626.

36. Pedersen BK, Saltin B. Evidence for prescribing exercise as therapy in chronic disease. Scand J Med Sci Sports 2006; 16 Suppl. 1: 3-63.

37. Frontera WR, Meredith CN, O'Reilly KP, KnuttGEN HG, Evans WJ. Strength conditioning in older men; skeletal muscle hypertrophy and improved function. J Appl Physiol 1988; 64: 1038-1044.

38. Treuth MA, Ryan AS, Pratley RE, Rubin MA, Miller JP, Nicklas BJ et al. Effects of strength training in total and regional body composition in older men. J Appl Physiol 1994; 77: 614-620.

39. HÄKKINEN K, HäKKINEN A. Neuromuscular adaptations during intensive strength training in middle-aged and elderly males and females. Electromyogr Clin Neurophysiol 1995; 35: 137-147.

40. HäKkinen K, Pakarinen A, Kraemer WJ, HÄKkinen A, Valkeinen H, Alen M. Selective muscle hypertrophy, changes in EMG and force, and serum hormones during strength training in older women. J Appl Physiol 2001; 91: 569580.

41. Häkkinen K, Alen M, Kallinen M, Newton RU, KRAEMER WJ. Neuromuscular adaptation during prolonged strength training and detraining, and re-strength training in middle-aged and elderly people. Eur J Appl Physiol 2000; 83: 51-62. 
42. Moritani T, De VRIEs H. Potential for gross muscle hypertrophy in older men. J Gerontol 1980; 35: 672-862.

43. Physical Activity Guidelines Advisory Committee. Report, 2008. Disponible en: http://www. hhs.gov/news/press/2008pres/10/20081007a. html (1 de noviembre, 2008).

44. American College of Sports Medicine Position Stand. The recommended quantity and quality of exercise for developing and maintaining cardiorespiratory and muscular fitness, and flexibility in healthy adults. Med Sci Sports Exerc 1998; 30: 975-991.

45. Kraemer WJ, Marchitelli L, Gordon SE, Harman E, Dziados JE, Mello R et al. Hormonal and growth factor responses to heavy resistance exercise protocols. J App Physiol( 1990;) 69: 1442-1450.

46. American College of Sport Medicine Position Stand. Exercise and physical Activity for older Adults. Med Sci Sports Exerc 1998; 30: 992-1008.

47. Izquierdo M, Ibañez J, Hakkinen K, Kraemer WJ, LARrión JL, Gorostiaga EM. Once weekly combined resistance and cardiovascular training in healthy older men. Med Sci Sports Exerc 2004; 36: 435-443.

48. Izquierdo-Gabarren M, González De Txabarri Expósito R, García-Pallarés J, Sánchez-Medina L, De VILLARREAl ES, IzQUIERdo M. Concurrent endurance and strength training not to failure optimizes performance gains. Med Sci Sports Exerc 2010; 42: 1191-1199.

49. Izquierdo M, González-Izal M, Navarro-AmezQueta I, CALbet JA, IbañEz J, Malanda A et al. Effects of strength training on muscle fatigue mapping from surface EMG and blood metabolites. Med Sci Sports Exerc 2011; 43: 303-311.

50. IZquierdo M, IBAÑEZ J, GonZÁLEZ-BAdILlo JJ, HÄKKINEN K, RATAmess NA, KraEmer et al. Differential effects of strength training leading to failure versus not to failure on hormonal responses, strength, and muscle power gains. J Appl Physiol 2006; 100: 1647-1656.

51. Steib S, Schoene D, Pfeifer K. Dose-response relationship of resistance training in older adults: a meta-analysis. Med Sci Sports Exerc 2010; 42: 902-914.

52. Tschopp M, SATtelmayer MK, HilfiKer R. Is power training or conventional resistance training better for function in elderly persons? A meta-analysis. Age Ageing 2011; 40: 549-556.

53. Clark DJ, Patten C, Reid KF, Carabello RJ, PhiLLIPS EM, FIELDING RA. Impaired voluntary neuromuscular activation limits muscle power in mobility-limited older adults. Gerontol A Biol Sci Med Sci 2010; 65: 495-502.

54. KaWAMORI, HafF GG. The optimal training load for the development of muscular power. J Strength Cond Res 2004; 18: 676-684.

55. Lemmer JT, Ivey FM, Ryan AS, Martel GF, HurlBUt DE, METTER JE et al. Effect of strength training on resting metabolic rate and physical activity: age and gender comparisons. Med Sci Sports Exerc 2001; 33: 532-541.

56. KRAEMER WJ, RATAMESS NA. Fundamentals of resistance training: progression and exercise prescription. Med Sci Sports Exerc 2004; 36: 674-688.

57. American Geriatrics Society Panel on Exercise and Osteoarthritis. Exercise prescription for older adults with osteoarthritis pain: consensus practice recommendations. A supplement to the AGS Clinical Practice Guidelines on the management of chronic pain in older adults. J Am Geriatr Soc 2001; 49; 808-823.

58. Williams MA, Haskell WL, Ades PA, Amsterdam EA, BitTner V, FrAnklin BA ET AL. American Heart Association Council on Clinical Cardiology; American Heart Association Council on Nutrition, Physical Activity, and Metabolism. Resistance exercise in individuals with and without cardiovascular disease: 2007 update: a scientific statement from the American Heart Association Council on Clinical Cardiology and Council on Nutrition, Physical Activity, and Metabolism. Circulation 2007 31; 116: 572-584.

59. LeVeritt M, Abernethy PJ, Barry BK y Logan PA. Concurrent strength and endurance training: a review. Sports Med 1999; 28: 413-427.

60. Kraemer WJ, Patton JF, Gordon SE, Harman EA, Deschenes MR, Reynolds K et al. Compatibility of high-intensity strength and endurance training on hormonal and skeletal muscle adaptations. J Appl Physiol 1995; 78: 976-989.

61. Binder EF, Brown M, Sinacore DR, Steger-May K, YARASHeski KE, Schechtman KB. Effects of extended outpatient rehabilitation after hip fracture: a randomized controlled trial. JAMA 2004; 292: 837-846.

62. Pahor M, Blair SN, Espeland M, Fielding R,Gill TM, GURALNIK JM et al. Effects of a physical activity intervention of measures of physical performance: Results of the lifestyle interventions and independence for elders Pilot (LIFE-P) study. J Gerontol A Biol Sci Med Sci 2006; 61: 1157-1165.

63. Chin A Paw MJ, van Uffelen JG, Riphagen I, van MEchelen W. The functional effects of a phy- 
sical exercise training in frail older people: a systematic review. Sports Med 2008; 38 : 781-793.

64. Daniels R, van Rossum E, de Witte L, Kempen GI, VAN DEN HEUvEL W Interventions to prevent disability infrail community-dwelling elderly: a systematic review. BMC Health Serv Res 2008 30; 8: 278.

65. Miller ME, Rejeski WJ, Reboussin BA, Ten Have TR, ETtinger WH. Physical activity, functional limitations and disability in older adults. J AM Geriatr Soc 2000; 48: 1264-1272.

66. Wu SC, LEU SY, LI CY. Incidence of and predictors for chronic disability in activities of daily living among older people in Taiwan. J Am Geriatr Soc 1999; 47: 1082-1086.

67. Peterson MJ, Giuliani C, Morey MC, Pieper CF, Evenson KR, Mercer V et al. Health, Aging and Body Composition Study Research Group. Physical activity as a preventative factor for frailty: the health, aging, and body composition study. J Gerontol A Biol Sci Med Sci 2009; 64: 61-68.

68. Panel on Prevent ion of Falls in Older Persons, American Geriatrics Society and British Geriatrics Society. Summary of the updated American geriatrics society/british geriatrics society clinical practice guideline for prevention of falls in older persons. J Am Geriatr Soc 2011; 59: 148-157.

69. Gillespie LD, Robertson MC, Gillespie WJ, Lamb SE, Gates S, Cumming RG et al. Interventions for preventing falls in older people living in the community. Cochrane Database Syst Rev 2009 : CD007146.

70. Gates S, Fisher JD, Cooke MW, Carter YH, Lamb SE. Multifactorial assessment and targeted intervention for prevention falls and injures among older people in community and emergency care settings: systematic review and meta-anaylisis. BMJ 2008; 336: 130-133.
71. García García J, Larrión Zugasti JL. Deterioro cognitivo y fragilidad. Jesús María Lopez Arrieta, Francisco José García Garcia, editores. El anciano con demencia. Sociedad Española de Medicina Geriátrica. Madrid, 2007: 59-83.

72. Liu-Ambrose T, Nagamatsu LS, Graf P, Beattie BL, Ashe MC, Handy TC. Resistance training and executive functions: a 12-month randomized controlled trial. Arch Intern Med 2010 25; 170: 170-178.

73. Casas Herrero A, Montero-Odasso M. Trastorno de la marcha y demencias. En: Leocadio Rodríguez Mañas, Roberto Petidier Torregrosa. Avances en demencia. Una perspectiva integral. Sociedad Española de Medicina Geriátrica. Madrid, 2010: 105-148.

74. Liu-Ambrose T, Davis JC, Nagamatsu LS, Hsu CL, Katarynych LA, Khan KM. Changes in executive functions and self-efficacy are independently associated with improved usual gait speed in older women. BMC Geriatr 2010; 10 : 25.

75. KATZ IR. Depression and frailty: the need for multidisciplinary research. Am J Geriatr Psychiatry 2004 ; 12: 1-6.

76. Bartholomew JB, Morrison D, Ciccolo JT. Effects of acute exercise on mood and well-being in patients with major depressive disorder. Med Sci Sports Exerc 2005; 37: 2032-2037.

77. Nicklas BJ, Hsu FC, Brinkley TJ, Church T, Goodpaster BH, KritchevsKy SB et al. Exercise training and plasma C-reactive protein and interleukin-6 in elderly people. J Am Geriatr Soc 2008; 56: 2045-2052.

78. Landi F, Abbatecola AM, Provinciali M, Corsonello A, Bustacchini S, Manigrasso L et al. Moving against frailty: does physical activity matter? Biogerontology 2010; 11: 537-545.

79. Hunter GR, McCarthy JP, Bamman MM. Effects of resistance training in older adults. Sports Med 2004; 34: 329-348. 
